\title{
Advertising, consensus, and ageing in multilayer Sznajd model
}

\author{
Christian Schulze \\ Institute for Theoretical Physics, Cologne University \\ D-50923 Köln, Euroland
}

November 11, 2018

e-mail: ab127@uni-koeln.de

\begin{abstract}
In the Sznajd consensus model on the square lattice, two people who agree in their opinions convince their neighbours of this opinion. We generalize it to many layers representing many age levels, and check if still a consensus among all layers is possible. Advertising sometimes but not always produces a consensus on the advertised opinion.

Keywords: Sociophysics, phase transition, ageing, Monte Carlo simulation

Many recent models tried to simulate how in a society a consensus may emerge [1, 2, 3, 4. Particularly well studied is the Sznajd model [5, 6] where two neighbouring people (on a one-dimensional chain or higher-dimensional lattice) convince their neighbours if and only if these two people agree in their opinions. We generalize here the square lattice to a multilayer model, where each layer correponds to a different age group of the simulated people. We check if the phase transition known for the simple square lattice is also valid in the multilayer model and ask how many different initial opinions are allowed if a consensus is still desired. Finally, we check the effects of advertising [7, 8, favouring the first of the several possible opinions.

Each site on an $L \times L$ square lattice has one of $m$ possible opinions, initially random. A pair of nearest neighbours, randomly selected in sequential
\end{abstract}




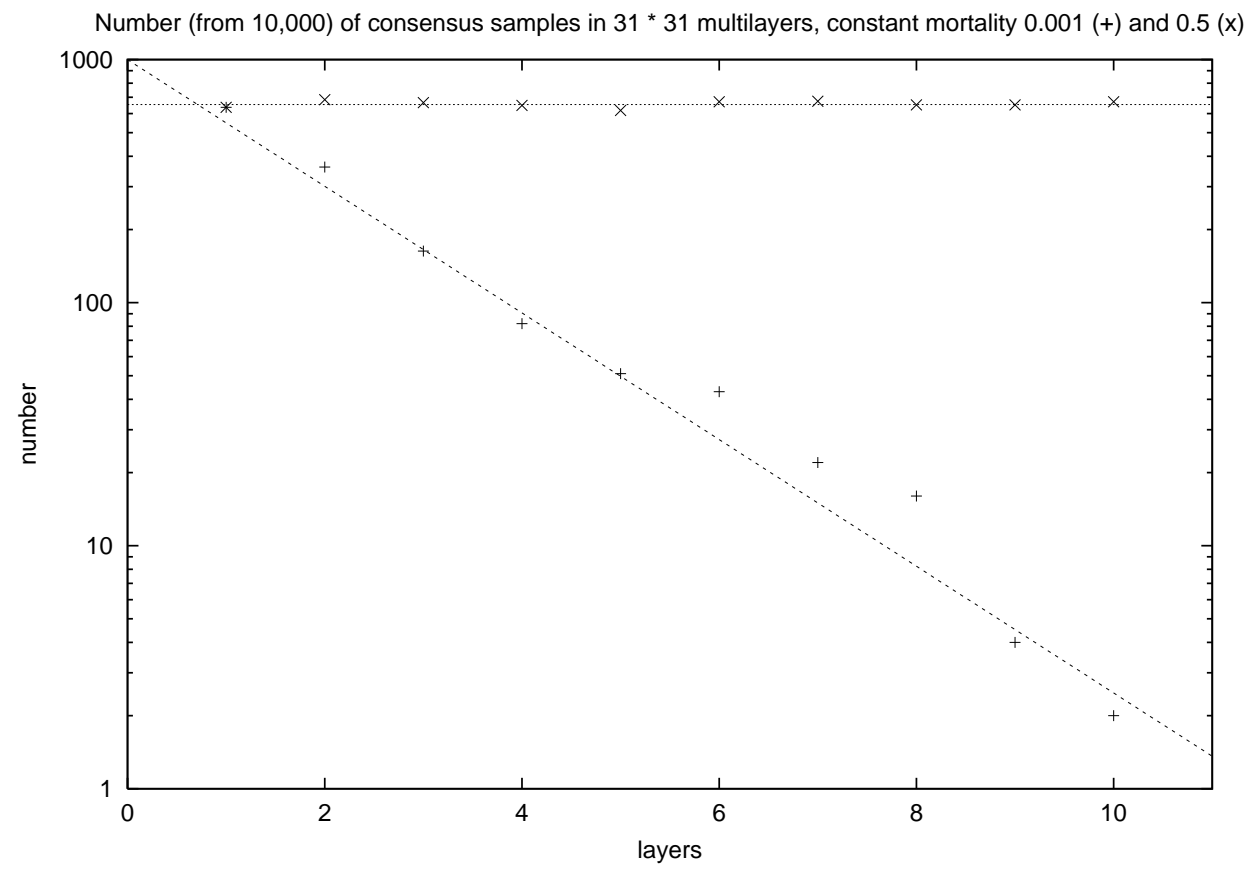

Figure 1: Number of samples, from $10^{4}$, where for $m=4$ a consensus is reached, versus the number $N$ of layers or age levels. For high mortalities the consensus probability stays at about $7 \%$ while at low mortalities it decays exponentially with $N$, nearly as $1 / 4^{N}$.

updating, may share the same opinion $j$. In this case, all six neighbours of this pair are convinced of that opinion, that means they are forced to adopt the same value $j$. This model has a phase transition [6] as a function of the initial concentration $p$ : If for $m=2$ the initial distribution of opinions is random and if the fraction $p$ of sites has the first and the remaining fraction $1-p$ the other opinion, then for large lattices everybody ends up with the first opinion if $p<1 / 2$ and everybody with the other opinion if $p>1 / 2$. If $m>2$ we assume bounded confidence [3, 2, which means that no neighbours can be convinced which differ by more than one opinion unit from the central pair. In that case [6] $m=3$ usually still leads to a consensus while $m=4$ seldomly allows a consensus: It is difficult to form a stable government coalition with four and more parties.

Now we generalize this planar Sznajd model to a multilayer model of $N$ 


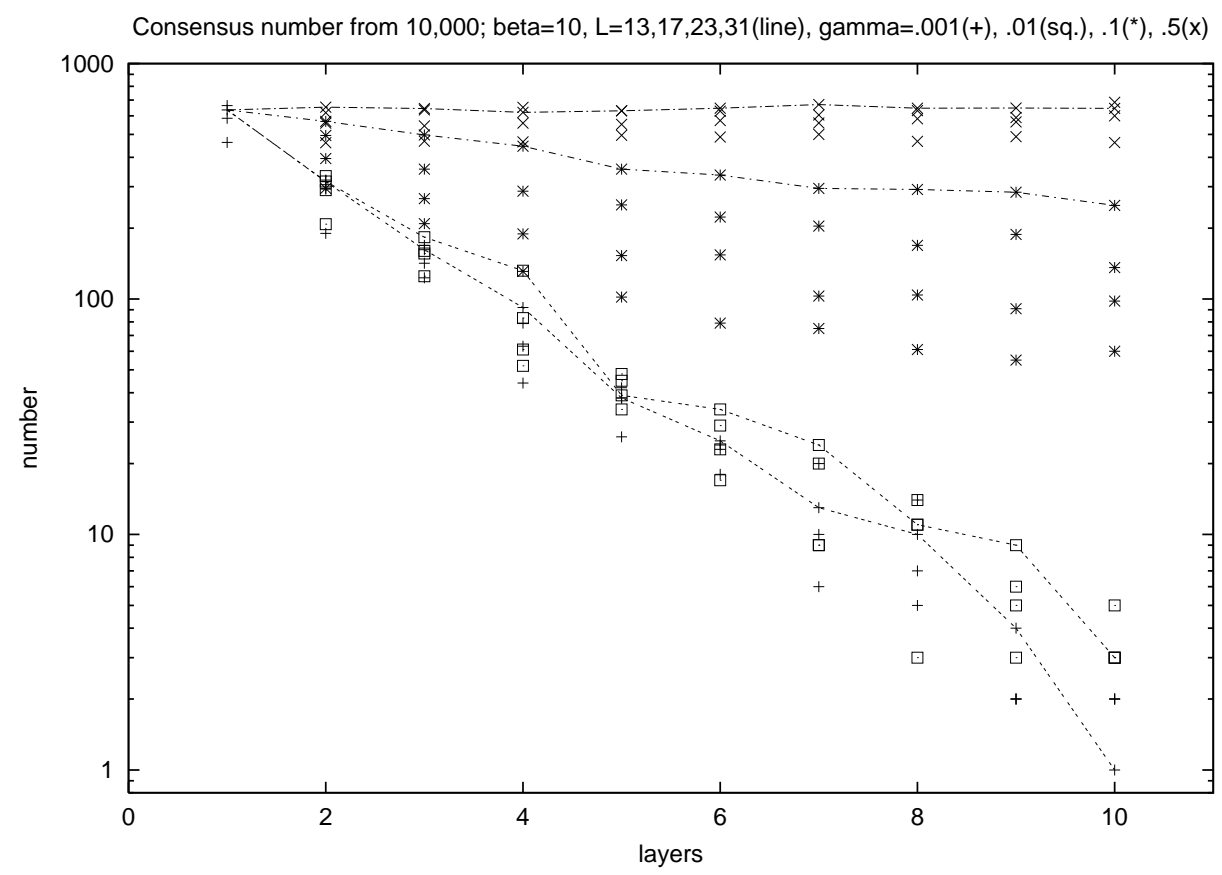

Figure 2: As Fig.1 but with ageing, for four mortality factors $\gamma=$ $1 / 10^{3}, 1 / 10^{2}, 1 / 10,1 / 2$ (from bottom to top) and four different lattice sizes $L=13,17,23,31$. The largest lattices are symbolized by lines.

square lattices on top of each other, representing $N$ age intervals in the life of an individual. With a certain probability $q$, called mortality, an individual dies, the younger ones on the same two-dimensional site all move up one time unit in their age, and a baby is born on the newly freed site, having the opinion of the parent. Within each layer only, and not between layers, the usual Sznajd convincing process takes place. First, we found that for two layers we still have a phase transition. Below we will check the chances of finding a consensus as a function of the number $N$ of layers in four cases: with an age-independent mortality (Fig.1), with a mortality increasing exponentially with age (Fig.2), with a probability to be convinced inversely proportional to mortality (Fig.3), and finally again with age-independent mortality and convincing probability but a constant advertising effort in favour of the first of $m$ opinions (Fig.4).

In this multilayer model we continue the simulations until the basic layer 


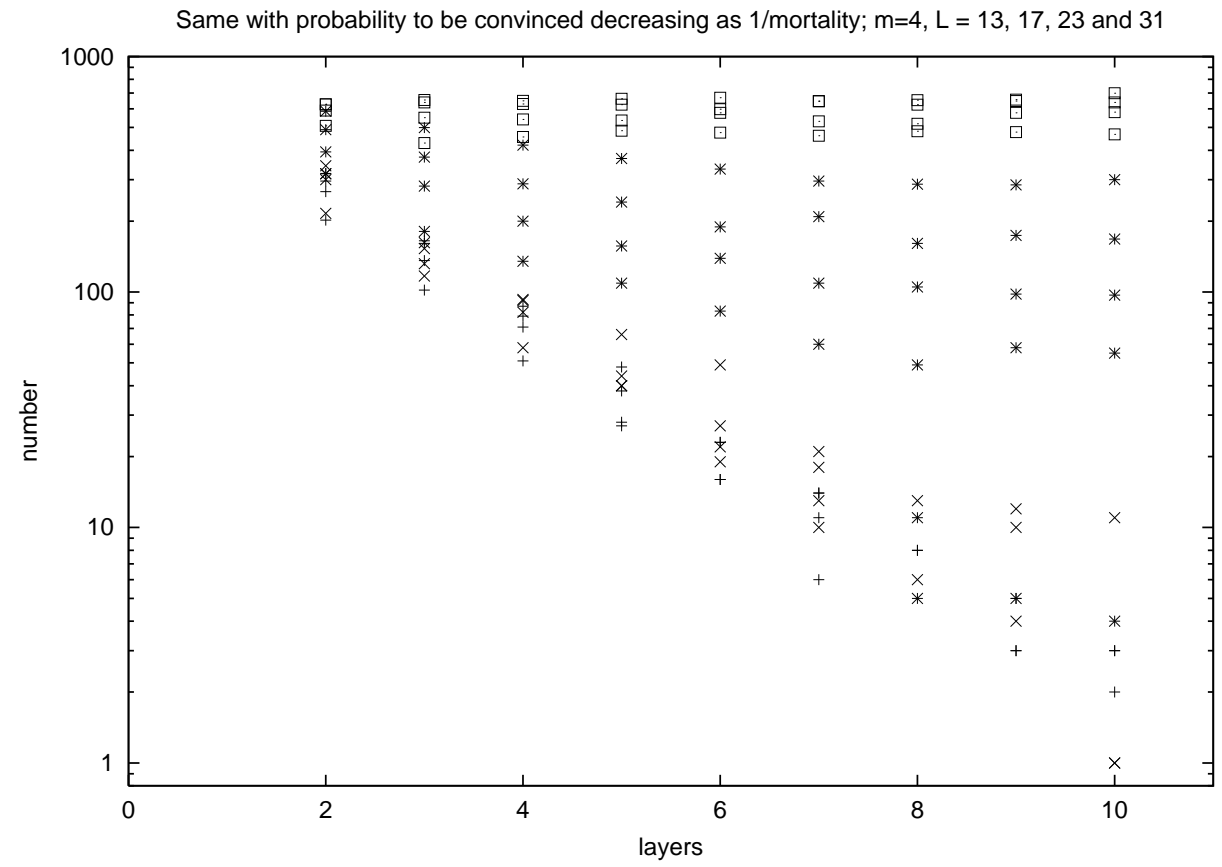

Figure 3: As Figs.1,2 but with convincing probability decreasing exponentially with age.

(the babies) reaches a complete consensus within that layer only. Then we find the majority opinion in each of the higher layers, and we declare a consensus if and only if the majority opinion in each of the higher layers agrees with the one surviving opinion in the basic layer.

With $m \leq 3$ possible opinions, a consensus is always formed for large lattices and long enough times, while for $m \geq 4$ a consensus is rare, just as for the single layer. We used $L=13,17,23,31,61$ and sometimes 101. Fig. 1 shows for a $31 \times 31$ lattice that for small mortalities $q=0.001$ the probability to reach a consensus, already below 7 percent for a single layer, decays exponentially towards zero with increasing number $N$ of layers. In contrast, for large mortalities, $q=0.5$, it stays at its single-layer value for all $2 \leq N \leq 10$. The higher mortality leads to a stronger coupling between layers and thus to easier overall consensus; note that no convincing takes place between different layers.

This trend of Fig.1 is well reproduced in Fig.2 if we include ageing, that 


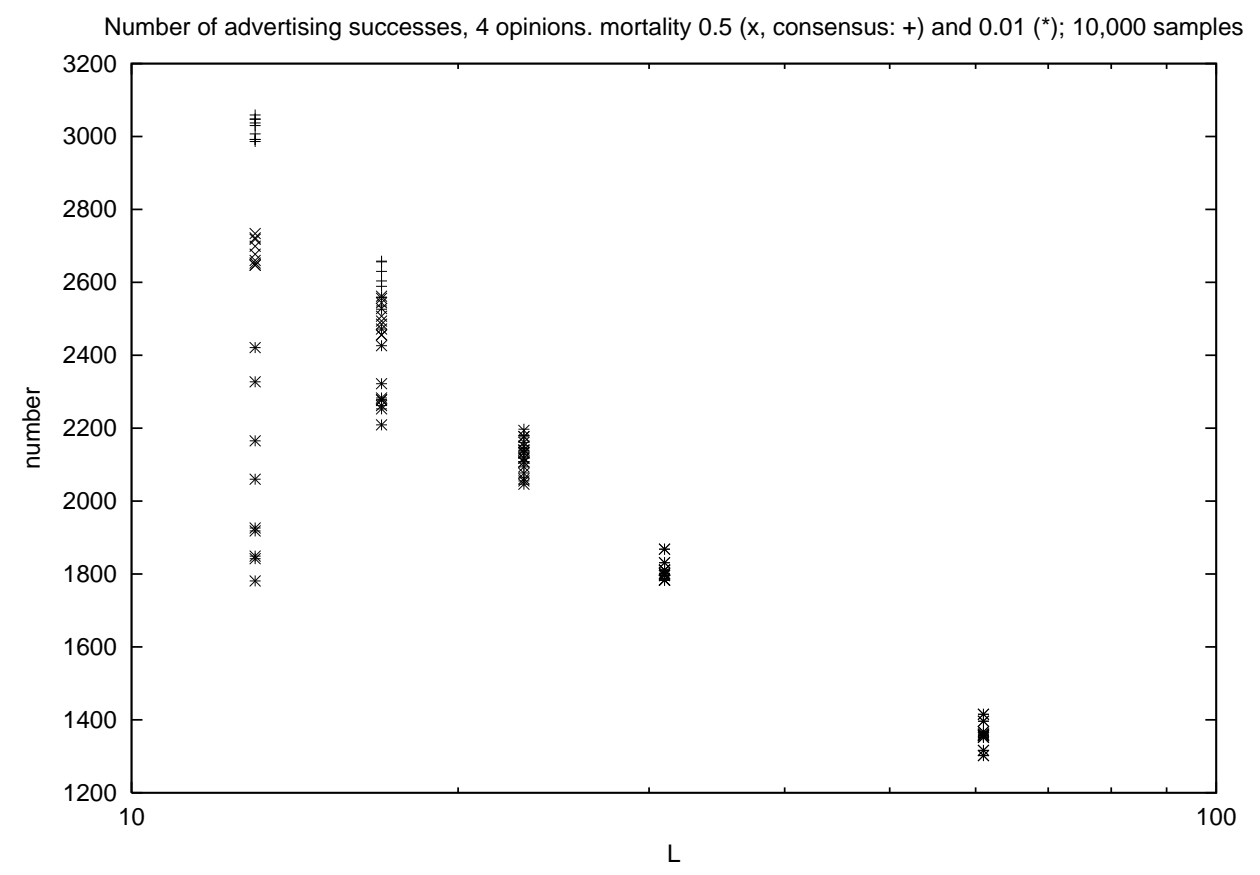

Figure 4: Number, among $10^{4}$ samples, of consensus $(+)$ and of success $\left(\mathrm{x},{ }^{*}\right)$ cases, for constant low $(*)$ and high $(+, \mathrm{x})$ mortality and $m=4$. A consensus is called a success if the consensus opinion is the advertised one. For low mortality nearly every consensus is a success and thus the consensus numbers are not shown.

means a mortality $q$ increasing exponentially with age $n=1,2, \ldots, N$ according to the Gompertz law of the 19th century: $q=\gamma \exp [10(n-N) /(N-1)]$. Not much changes if we include that young people are more easily convinced than old people and thus take a convincing probability $\exp [10(N-n) /(N-$ $1)]=\gamma / q$ in Fig. 3 .

Advertising has the effect that independent of the whole convincing process, a site assumes the first opinion with probability 0.1 , at every iteration. The mortality is taken again as 0.01 independent of age, and convincing always takes place. For $m=2$ opinions, advertising always wins for large enough lattices, just as in a single layer [7, 8]; also for $m=3$ advertising reaches a complete consensus for the advertised opinion. Fig. 4 shows a more complicated behaviour for $m=4$ : Now with increasing lattice size the 
fraction of advertising successes shrinks, possibly towards zero. It is quite independent of the number of layers which varies from 2 to 10. Fig.4 for small lattices like $L=13$ also shows that sometimes a consensus is reached but not for the advertised opinion.

In summary, the multilayer model is similar to the single-layer Sznajd model in allowing a consensus for two and three opinions but not for four (and more). This effect remains if advertising, a mortality increasing with age, and a convincing probability decreasing with age are introduced. The low probability of consensus, in the case of four opinions without advertising, decreases exponentially with the number of age layers.

Thanks are due to D. Stauffer for help.

\section{References}

[1] R. Axelrod, The Complexity of Cooperation, Princeton University Press 1997

[2] G. Weisbuch, G. Deffuant, F. Amblard, and J.-P. Nadal, Journal of Artificial Societies and Social Simulation 5, No. 4, paper 1 (2002) (electronic only through jasss.soc.surrey.ac.uk).

[3] R. Hegselmann and M. Krause, Journal of Artificial Societies and Social Simulation 5, No.3, paper 2 (2002).

[4] E. Ben-Naim, P.L. Krapivsky and S. Redner, Physica D 183, 190 (2002).

[5] K. Sznajd-Weron and J. Sznajd, Int. J. Mod. Phys. C 11, 1157 (2000)

[6] D. Stauffer, Journal of Artificial Societies and Social Simulation 5, No. 1, paper 4 (2000) and AIP Conference Proceedings 690, 147 (2003).

[7] C. Schulze, Int. J. Mod. Phys. C 14, 95 (2003)

[8] K. Sznajd-Weron and J. Sznajd, Physica A 324, 437 (2003) 